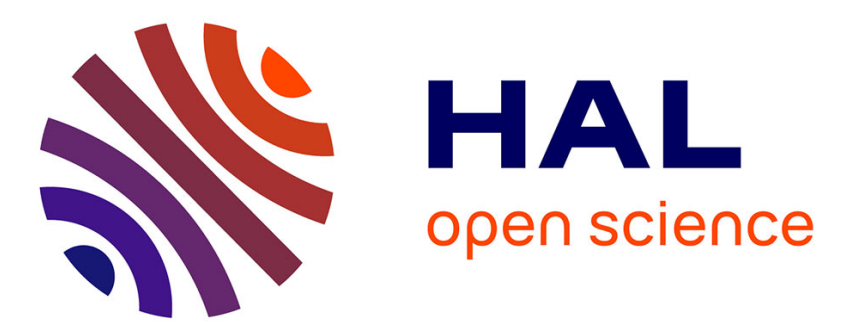

\title{
Doherty Amplifier Optimization Using Robust Genetic Algorithm and Unscented Transform
}

Marcos Carneiro, Paulo H. P. de Carvalho, Nathalie Deltimple, Leonardo da

C. Brito, Leonardo Rax de Menezes, Eric Kerherve, Sergio G. de Araujo,

Adson S. Rocha

\section{To cite this version:}

Marcos Carneiro, Paulo H. P. de Carvalho, Nathalie Deltimple, Leonardo da C. Brito, Leonardo Rax de Menezes, et al.. Doherty Amplifier Optimization Using Robust Genetic Algorithm and Unscented Transform. IEEE International NEWCAS'2011, Jun 2011, Bordeaux, France. pp.4. hal-00669875

\section{HAL Id: hal-00669875 https://hal.science/hal-00669875}

Submitted on 14 Feb 2012

HAL is a multi-disciplinary open access archive for the deposit and dissemination of scientific research documents, whether they are published or not. The documents may come from teaching and research institutions in France or abroad, or from public or private research centers.
L'archive ouverte pluridisciplinaire HAL, est destinée au dépôt et à la diffusion de documents scientifiques de niveau recherche, publiés ou non, émanant des établissements d'enseignement et de recherche français ou étrangers, des laboratoires publics ou privés. 


\title{
Doherty Amplifier Optimization Using Robust Genetic Algorithm and Unscented Transform
}

\author{
Marcos L. Carneiro*, Paulo H. P. de Carvalho ${ }^{\dagger}$, Nathalie Deltimple , Leonardo da C. Brito $^{\S}$, \\ Leonardo R.A.X. de Menezes "Eric Kerherve", Sergio G. de Araujo** and Adson S. Rocha ${ }^{\dagger \dagger}$ \\ Electrical Engineering Department, University of Brasilia Brazil, Brasilia \\ Email: *marcoslajovic@unb.br, ${ }^{\dagger}$ paulo@ene.unb.br, ${ }^{\dagger \dagger}$ adsonrocha@gmail.com, $\uparrow_{\text {leonardo@ene.unb.br }}$ \\ University of Bordeaux, Bordeaux, France \\ Email: ${ }^{\ddagger}$ nathalie.deltimple@ims-bordeaux.fr, $\|_{\text {eric.kerherve@ims-bordeaux.fr }}$ \\ Electrical Engineering Department, Federal University of Goias, Goiania, Brazil \\ Email: ${ }^{\S}$ brito@eee.ufg.br, ${ }^{* *}$ granato@eee.ufg.br
}

\begin{abstract}
A robust genetic circuit optimizer using Unscented Transform and Non-dominated Sorting Genetic Algorithm-II is presented. The algorithm provides significant decrease in computational cost compared to Monte Carlo method. This transform permits the circuit performance uncertainties determination from components uncertainties, thus, a search through robustness can be done. Results shows reduced computational costs, the many possibilities provided to circuit designer by the multi-objective search and assumptions that can be done in a Doherty power amplifier study with the optimizer.
\end{abstract}

\section{INTRODUCTION}

Implementation process have inherent tolerances and limitations that always make the final product to have different performance from the project. Electronic components have tolerance ranges that results also in a uncertainty goals range. To build robust circuits, it's necessary to reduce the possible performance range variation, making it less sensible as possible according to components uncertainties.

Despite the fact of all circuits behavior been equation modeled, the presence of parasitics difficult the project and even makes impossible an analytic optimization. The local minimal problem in non-linear circuits makes gradient based optimization to get stuck [1], so stochastic optimization techniques are growing and giving remarkable results [2]-[5].

The uncertainty analysis makes the computational cost a problem. Monte Carlo method is precise for many iterations but it makes the time simulation cost unfeasible. Unscented Transform (UT) [6], [7] arises as a promising technique to cope this problem.

This research shows the union of UT and Non-dominated Sorting Genetic Algorithm-II (NSGA-II) to give circuit designers an optimization tool to better meet multi-objective problems, robust circuits, get results in feasible time and to carefully analyze all possible best circuits behavior.

This paper starts the presentation of genetic algorithm NSGA-II, in section II, discussing non-dominant concept and population evolution process. In section III, a brief explanation of UT is done, then, in section IV, UT method for circuit performance uncertainty is presented. Next, in section $\mathrm{V}$, the optimizer methodology shows using UT in conjunction to NSGA-II. Section VI, presents the results and their analysis are done in section VII. The paper is concluded in section VIII and finally in section IX some remarks to future works are done.

\section{NSGA-II}

NSGA-II is a non-domination based genetic algorithm for multi-objective optimization [8]. It is a very effective algorithm with elitism, low complexity and with better sorting algorithm than last NSGA [8], [9].

Once the population is initialized, each individual (circuit) is simulated and a fitness value is assigned to each objective function. Based in the non-domination concept, individuals are placed into each Pareto's Front.

The first front is a completely non-dominant set in the current population and the second front being dominated by the individuals in the first front only, and next fronts follows the same concept. A rank number is assigned to each front for posterior individuals selection. Individuals in the top most front has rank number 1 , others in the second has rank number 2 and so on.

In addition to fitness value there is a parameter called crowding distance. It's a measure of how close an individual is to its neighbors. Large crowding distance results in better diversity in the population, so the multi-objective plane is homogeneous covered.

Individuals are selected for reproduction using binary tournament selection (SBX) crossover [10], [11] and polynomial mutation [11], [12] based on the rank and crowding distance. Individuals are selected if they have inferior rank number (superior fronts) or if crowding distance is greater than the other. The selected population generates offsprings from crossover and mutation operators. Parent population and offspring population are sorted again based on non-domination and only $\mathrm{N}$ individuals (population size) are selected to the next generation. 


\section{UNSCENTED TRANSFORM}

The UT is a method to compute random variables statistics that passes a non-linear transformation [6], [7]. The UT uses a group of selected points (sigma points) and weights to approximate a non-linear mapping.

The sigma points are fixed values inside the random variable distribution. These fixed points passes through non-linear transformation and final values are multiplied by weights. With the transformed sigma points, the statistics of mean and variance are computed.

The problem can be defined by an n-dimensional random variable $\mathrm{x}$ with a defined probability distribution function, with mean $\bar{x}$, co-variance $P_{x x}$, that passes a non-linear transformation $y=g(x)$ [7], [13], [14], [16]. It's desired an approximation for the mean $\bar{y}$ and covariance $P_{y y}$ for $\mathrm{y}$ random variable. From that transformation, it is possible to get moments approximation of a continuous probability distribution $w(\hat{u})$ from the moments of a discrete distribution $w_{i}$. Thus, UT makes a discrete distribution with the same moments of a continuous distribution after transformation. This equality is showed in equation 1 :

$$
E_{d}\left\{\hat{u}^{k}\right\}=\int_{-\infty}^{\infty} \hat{u}^{k} \omega(\hat{u}) d \hat{u}=\sum_{i} \omega_{i} S_{i}^{k}
$$

Where $\hat{u}$ is the random variables set with known distribution, $w(\hat{u})$ the continuous probability distribution function, $w_{i}$ the discrete probability distribution function, $S_{i}$ the sigma points and $\mathrm{k}$ the approximation order. After sigma points and weights determination, the mean of $g(x), \bar{G}$, is computed in equation 2:

$$
\bar{G}=E\{G(\bar{U}+\hat{u})\}=\sum_{i} \omega_{i} G\left(\bar{U}+S_{i}\right)
$$

Variance of $g(x)$ is computed in equation 3:

$$
E\left\{(G(\bar{U}+\hat{u})-\bar{G})^{2}\right\}=\sum_{i} \omega_{i}\left(G\left(\bar{U}+S_{i}\right)-\bar{G}\right)^{2}
$$

The distribution moments computation problem can be handle by Monte Carlo method. UT is a new resolution approach used for intensive search methods with high cost functions, where the minimum number of simulations is needed.

\section{UNSCENTED TRANSFORM APPLICATION AND COST REDUCTION ANALYSIS}

To analyze the cost reduction, UT was applied and a comparison with the Monte Carlo method was done. The power-added efficiency (PAE) of a Doherty power amplifier (Fig.1) was used as function $y=g(x)$.

For these preliminary research results, a 1000 iteration Monte Carlo was considered the most precise result and it was used for base comparing (Table I) to a UT with four random variables $(x=[x 1, x 2, x 3, x 4])$, with Taylor series truncation in the second term. With these specifications, the sigma points were computed with technique presented in [13] resulting in the need of 25 simulations [15].
Table II shows the computed sigma points and it's weights. Method in [13] makes the first sigma point to have an almost insignificant weight but it was maintained in the table for showing the number of produced points with the technique.

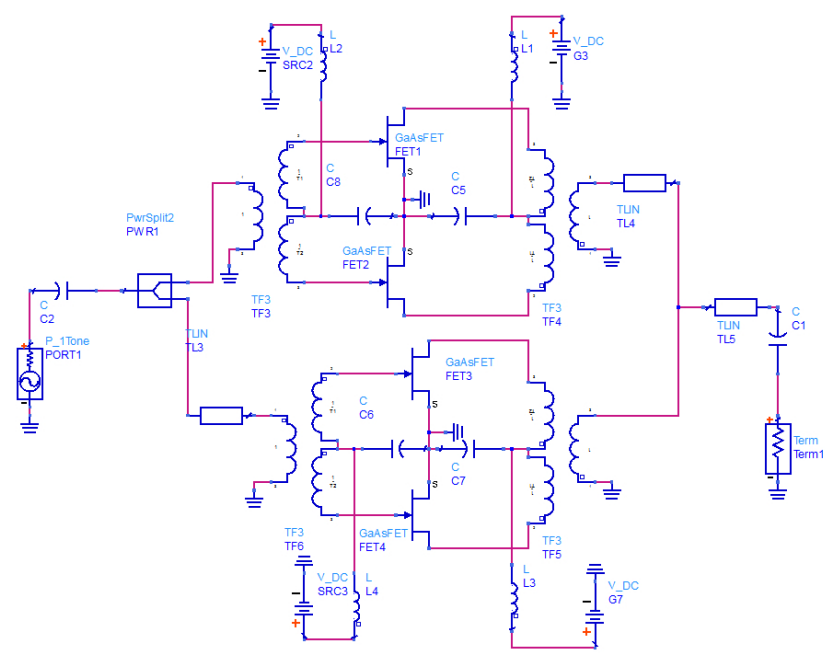

Fig. 1. Optimized schematic

TABLE I

Monte Carlo and UT comparisons with a Monte Carlo 1000 ITERATION

\begin{tabular}{|r|r|r|r|}
\hline & & Max Error(\%) & Min Error (\%) \\
\hline Mean & Monte Carlo 500 & 1.422621426 & 0.052105018 \\
& Monte Carlo 100 & 2.633516331 & 0.07266602 \\
& Monte Carlo 25 & 6.75630089 & 0.480405393 \\
& UT 25 & 0.786531344 & 0.027630973 \\
\hline Std. dev. & Monte Carlo 500 & 3.307979041 & 0.263539783 \\
& Monte Carlo 100 & 13.88537443 & 0.289326638 \\
& Monte Carlo 25 & 21.30773821 & 3.867651622 \\
& UT 25 & 3.249667183 & 0.054493952 \\
\hline
\end{tabular}

The used circuit was a Doherty Lite power amplifier [17] with transistors in push-pull configuration in each amplifier (Fig.1). The first two transistors in Fig.1 make the main amplifier and the last two, the peak amplifier. The four chosen circuit random variables were the main amplifier gate bias voltage SRC2 $(x 1)$, the peak amplifier bias gate voltage SRC3 $(x 2)$, the transmission line characteristic impedance TLIN3 before peak amplifier $(x 3)$ and the transmission line TLIN5 characteristic impedance before the load (load matching network - $x 4$ ). All four variables were modeled with normal distribution and with a 5\% tolerance from its nominal value.

Only four variables were chosen by hardware speed limitations given that an UT to five variables needs 45 simulations for each individual in the population, for each generation. Results for more optimization variables goes to future work. The specific circuit variables were chosen for been considered the most sensible for Doherty performance [17].

All simulations were done in the Agilent Advanced Design System, and for showing the computational cost reduction, comparisons were done with 500, 100 and 25 Monte Carlo iterations. 
TABLE II

SIGMA POINTS AND WEIGHTS TO A FOUR VARIABLE UT

\begin{tabular}{|c|c|c|c|c|c|}
\hline & \multicolumn{4}{|c|}{ Sigma Points } & Weights \\
\hline 1 & 0 & 0 & 0 & 0 & 0 \\
\hline 2 & -1.7321 & -1.7321 & -1.7321 & -1.7321 & 0.0069 \\
\hline 3 & 1.7321 & -1.7321 & -1.7321 & -1.7321 & 0.0069 \\
\hline 4 & -1.7321 & 1.7321 & -1.7321 & -1.7321 & 0.0069 \\
\hline 5 & 1.7321 & 1.7321 & -1.7321 & -1.7321 & 0.0069 \\
\hline 6 & -1.7321 & -1.7321 & 1.7321 & -1.7321 & 0.0069 \\
\hline 7 & 1.7321 & -1.7321 & 1.7321 & -1.7321 & 0.0069 \\
\hline 8 & -1.7321 & 1.7321 & 1.7321 & -1.7321 & 0.0069 \\
\hline 9 & 1.7321 & 1.7321 & 1.7321 & -1.7321 & 0.0069 \\
\hline 10 & -1.7321 & -1.7321 & -1.7321 & 1.7321 & 0.0069 \\
\hline 11 & 1.7321 & -1.7321 & -1.7321 & 1.7321 & 0.0069 \\
\hline 12 & -1.7321 & 1.7321 & -1.7321 & 1.7321 & 0.0069 \\
\hline 13 & 1.7321 & 1.7321 & -1.7321 & 1.7321 & 0.0069 \\
\hline 14 & -1.7321 & -1.7321 & 1.7321 & 1.7321 & 0.0069 \\
\hline 15 & 1.7321 & -1.7321 & 1.7321 & 1.7321 & 0.0069 \\
\hline 16 & -1.7321 & 1.7321 & 1.7321 & 1.7321 & 0.0069 \\
\hline 17 & 1.7321 & 1.7321 & 1.7321 & 1.7321 & 0.0069 \\
\hline 18 & 1.7321 & 0 & 0 & 0 & 0.1111 \\
\hline 19 & 0 & 1.7321 & 0 & 0 & 0.1111 \\
\hline 20 & 0 & 0 & 1.7321 & 0 & 0.1111 \\
\hline 21 & 0 & 0 & 0 & 1.7321 & 0.1111 \\
\hline 22 & -1.7321 & 0 & 0 & 0 & 0.1111 \\
\hline 23 & 0 & -1.7321 & 0 & 0 & 0.1111 \\
\hline 24 & 0 & 0 & -1.7321 & 0 & 0.1111 \\
\hline 25 & 0 & 0 & 0 & -1.7321 & 0.1111 \\
\hline
\end{tabular}

Results show that with only 25 simulations, the UT was capable to get better approximation than all other Monte Carlo tests for mean and standard deviation. Fig.3 shows the circuit power-added efficiency (PAE) uncertainty caused by $5 \%$ tolerance in each normal random variable, computed by UT. The PAE graph was computed with a power sweep simulation, so the uncertainties were done for each output power point. Performance variations were obtained along the graph, so, Table I shows the maximum and the mininum computed error.

\section{Circuit Optimization Method}

The proposed circuit optimization method is applied to a prior projected circuit. Next, components for optimization are chosen and their uncertainties are modeled by defining its mean and standard deviation for a normal distribution shape. Finally, a range variation for its mean is defined. The parameters set to guide the NSGA-II are the number of individuals, the max generation number (stop criterion), the multi-objective function, cross-over and mutation percentage.

For presented results in this study, four variables were chosen for optimizing (the same presented in section above). Thus, 25 additional simulations are done for each individual in the population to determine the possible variation in final performance parameters (robustness parameter).The optimization was done with 20 individual population, 20 maximum generations, $90 \%$ cross-over and $10 \%$ mutation. So the entire process take 10400 simulations (20x20x26). The final population is compound by 20 individuals in the Pareto's Front, each having maximum crowding distance and not dominated by none of all other individuals.

\section{Results}

A Doherty power amplifier was optimized and search goals were applied in the PAE backoff region [17]. Three objectives were defined for this optimization: mean PAE increasing, backoff range increasing and robustness increasing (standard deviation decreasing). To compare the results, a classic search was done (search without the robustness parameter).

The PAE performance parameter for all circuits in each population were plotted to give a vision of all possibilities in the Pareto's Front and to see the robustness parameter acting in modifying the search. Fig.2 shows the robust and classic optimization PAE population performance. Circuits with higher backoff in the two optimizations are shown in Fig. 3 and compared in Table III.

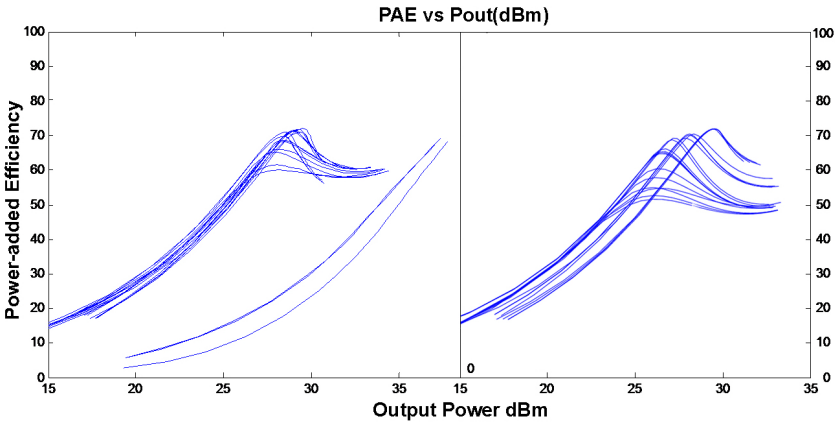

Fig. 2. PAE population performance for robust (left) and classic (right) optimization

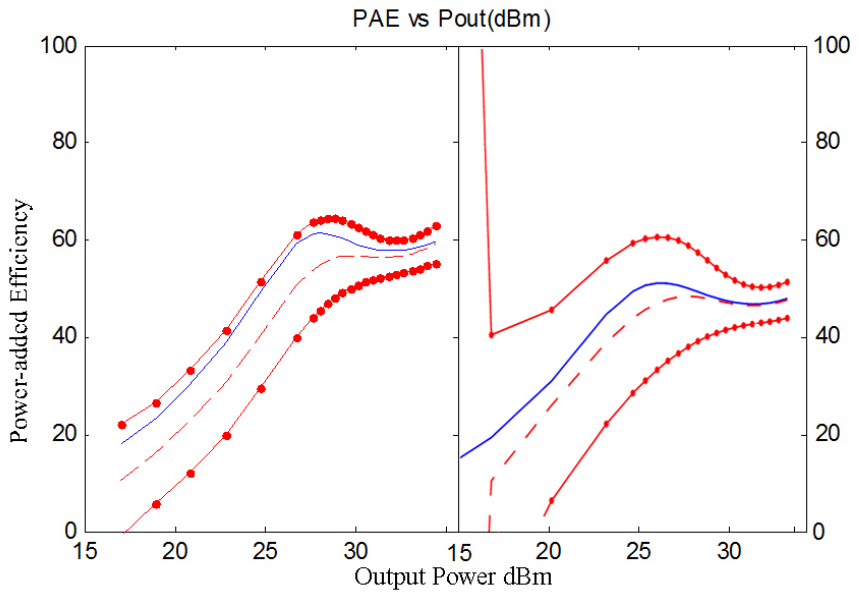

Fig. 3. Circuits with higher backoff from robust (left) and classic (right) optimization

\section{Result Analyses}

Comparing individuals in each optimization type in Fig.2, a first observation is the number of circuits with Doherty PAE behavior. The robust optimization showed three graphs without the constant PAE region and all circuits in classic optimization showed this characteristic. This result were reached because with the only four optimized variables, a greater robustness 
could be founded in a behavior without the Doherty loadpulling, and three circuits with Class $\mathrm{AB} / \mathrm{B}$ behavior have gone to Pareto's Front.

Another note is that the robustness parameter has concentrated the results in Fig.2 (left). The classic search is blind for the robust parameter, so, all circuits covered almost equally spaced the PAE possible region. This indicates that there is a link between the efficiency and robustness. Some projected efficiency shapes are less probable to yield success in the fabrication.

The robust search also shows efficiency for all circuits near $60 \%$ or more. The classic search shows results with constant efficiency region starting from $45 \%$. Thus, in the left part of Fig.2, circuits with higher robustness and efficiency were obtained.

The two circuits with higher backoff in each optimization population are shown in Fig. 3 and a clear difference in robustness can be seen. Table III compares these two circuits. The robust search have got a circuit with less backoff but with more than $10 \%$ more mean efficiency in the backoff region. The less standard deviation also shows its better robustness.

TABLE III

COMPARING RoBUST AND ClASSIC OPTIMIZATION

\begin{tabular}{|r|r|r|}
\hline & Robust Optimization & Classic Optimization \\
\hline PAE & 59.54552 & 49.442863 \\
Backoff & 6.3979183 & 7.1720743 \\
Std. dev. & 5.2832879 & 6.9888298 \\
\hline
\end{tabular}

\section{CONCLUSION}

The UT technique was presented to compute the circuit performance uncertainties based in its components uncertainties. Results were also validated with Monte Carlo method with 1000 iterations and comparisons were done. The demonstrated cost reduction allowed the association of UT and NSGA-II to create an optimizer guided by robustness.

This robust UT optimizer make a scan in the circuit performance possibilities inside variables defined range. The search guided by robustness parameter permits to study the circuit behavior and to define circuits with better chances to been successful implemented. This ability aides the circuit designer to meet multi-restrictions, measure possible variations and to avoid unexpected results from fabrication process.

\section{Perspectives for future WOrks}

Future works aims to collect data for different components standard deviations, probability distributions and to insert component parasitics and more optimization variables, like, transistors size and number of Doherty stages.

\section{ACKNOWLEDGMENT}

The authors would like to thank the financial support from University of Brasilia Electrical Engineering Department and from CAPES/COFECUB program.

\section{REFERENCES}

[1] J.W. Bandler and S.H. Chen, Circuit optimization: the state of the art IEEE Trans. Microwave Theory Tech., vol. MTT-36, n2, pags.424-443, 1988.

[2] R. Phelps, et al., Anaconda: simulation-based synthesis of analog circuits via stochastic pattern search. IEEE Trans. Computer-aided Design of Integr. Circuits and Systems, vol. 19, n6, pags.703-717, 2000.

[3] J. Silva and N. Horta, Genom: circuit-level optimizer based on a modified Genetic Algorithm kernel. Proc. Int. Symp. on Circuits and Systems, 2002.

[4] N. Paulino, J. Goes and A. Steiger-Gracao, Design methodology of optimization of analog building blocks using Genetic Algorithms. IEEE Int. Symp. on Circuits and Systems, 2001.

[5] L.C. Brito, P.H.P. de Carvalho, L.A. Bermudez, Multi-objective evolutionary optimisation of microwave oscillators. Electronics Letters, v.40, n.11, p. 1-2, 2004.

[6] S. Julier and J.K. Ulmann, General method for approximating nonlinear transformations of probability distributions, Eng. Dept. Univ. Oxford, Tech. Report., Oxford, 1996.

[7] L. de Menezes et al., Efficient extraction of statistical moments in electromagnetic problems solved with the method of moments, Microwave and Optoelectronics Conference, IMOC 2007. SBMO/IEEE MTT-S International, p.757-760, 2007.

[8] D. Kalyanmoy, A. Pratap, S. Agarwal and T. Meyarivan, A Fast Elitist Multi-objective Genetic Algorithm: NSGA-II, IEEE Transactions on Evolutionary Computation, 6(2):182-197, April 2002.

[9] N. Srinivas and D. Kalyanmoy, Multiobjective Optimization Using Nondominated Sorting in Genetic Algorithms, Evolutionary Computation, 2(3):221-248, 1994

[10] B. Hans-Georg and D. Kalyanmoy, On Self-Adaptive Features in RealParameter Evolutionary Algorithm, IEEE Transactions on Evolutionary Computation, 5(3):250-270, June 2001.

[11] D. Kalyanmoy and R.B. Agarwal, Simulated Binary Crossover for Continuous Search Space, Compex Systems, 9:115-148, April 1995.

[12] M.M. Raghuwanshi and O.G. Kakde, Survey on multiobjective evolutionary and real codded genetic algorithms. In Proceedings of the 8th Asia Pacific Symposium on Intelligent and Evolutionary Systems, pages 150-161, 2004.

[13] L. de Menezes et al., Efficient computation of stochastic electromagnetic problems using unscented transforms, Science, Measurement and Technology, IET, v.2, n.2, p.88-95, March, 2008.

[14] L. de Menezes et al., Modeling device manufacturing uncertainty in electromagnetic simulations, Microwave Symposium Digest, 2008 IEEE MTT-S International, p.1385-1388, June 2008.

[15] L. de Menezes et al., A new procedure for assessing the sensitivity of antennas using the Unscented Transform, IEEE Transactions on antennas and propagation, vol.58, no.3, march 2010.

[16] J. Pereira, L. de Menezes and G. Borges, Statistical analysis of induced ground voltage using the TLM+UT method, Electromagnetic Compatibility, EMC 2008, p.1-4, Aug. 2008.

[17] S.C. Cripps, $R F$ power amplifiers for wireless communications, 2nd ed., Artech House microwave library, 2006. 